\title{
Placebo-Controlled Clinical Trial of Incobotulinumtoxin A for Sialorrhea in Children
}

\section{SIPEXI}

Steffen Berweck, MD, Marcin Bonikowski, MD, PhD, Heakyung Kim, MD, Michael Althaus, MD, FFPM, Birgit Flatau-Baqué, Daniela Mueller, PhD, and Marta Dagmara Banach, MD, PhD

Neurology ${ }^{\circledR}$ 2021;97:e1425-e1436. doi:10.1212/WNL.0000000000012573

\section{Abstract}

\section{Background and Objectives}

To investigate the efficacy and safety of repeated injections of incobotulinumtoxinA (incoBoNT/ A) for treatment of chronic sialorrhea (drooling) associated with neurologic disorders (e.g., cerebral palsy, traumatic brain injury) or intellectual disability in children and adolescents in a prospective phase III study (SIPEXI [Sialorrhea Pediatric Xeomin Investigation]).

\section{Methods}

The study enrolled 2- to 17-year-old patients with sialorrhea due to neurologic disorders or intellectual disability. Patients received body weight-dependent doses of incoBoNT/A (20-75 U). A main period with 1 injection cycle (placebo-controlled, double-blind, 6- to 17-year-olds) was followed by an open-label extension with up to 3 further cycles. An additional cohort of 2- to 5-yearolds received active treatment throughout the study. Coprimary endpoints were the change in unstimulated salivary flow rate (uSFR) from baseline to week 4 and the carers' Global Impression of Change Scale (GICS) rating at week 4. Adverse events were recorded.

\section{Results}

In the main period, 220 patients aged 6-17 years were randomized and treated (148 patients in incoBoNT/A group, 72 patients in placebo group). A total of 35 patients aged $2-5$ years received incoBoNT/A (no placebo). A total of 214 patients aged 6-17 years and 33 patients aged 2-5 years continued treatment in the open-label extension period. For the 6- to 17-yearolds, a significant difference between incoBoNT/A and placebo was seen in mean uSFR decrease (difference $-0.06 \mathrm{~g} / \mathrm{min} ; p=0.0012$ ) and the carers' GICS rating (difference 0.28 points; $p=0.032$ ) at week 4 , in favor of active treatment. The secondary endpoints consistently supported these results. A sustained benefit was observed during the extension. Incidences of adverse events were comparable between incoBoNT/A and placebo and did not increase notably with repeated injections. The most common adverse events were respiratory infections. Efficacy and safety were also favorable in the uncontrolled cohort of 2- to 5-year-olds.

\section{Discussion}

Both co-primary efficacy endpoints were reached and superiority of incoBoNT/A over placebo was confirmed. IncoBoNT/A (up to $75 \mathrm{U}$, up to 4 cycles) is an effective and well-tolerated treatment for sialorrhea associated with neurologic disorders in children.

\author{
Correspondence \\ Dr. Berweck \\ sberweck@schoen-klinik.de
}

MORE ONLINE

III Class of Evidence

Criteria for rating

therapeutic and diagnostic

studies

NPub.org/coe

\section{CME Course}

NPub.org/cmelist

\footnotetext{
From the Specialist Center for Paediatric Neurology, Neurorehabilitation and Epileptology (S.B.), Schoen Klinik, Vogtareuth; Department of Pediatric Neurology and Developmental Medicine (S.B.), LMU Center for Children with Medical Complexity-Integrated Social Pediatric Center, Dr. von Hauner Children's Hospital, Ludwig-Maximilians-University, Munich, Germany; Movement Analysis Lab, Neuro Rehabilitation Department (M.B.), Mazovian Neuropsychiatry Center LLC, Warsaw, Poland; Pediatric Rehabilitation Medicine at the Department of Rehabilitation \& Regenerative Medicine (H.K.), Columbia University Irving Medical Center/New York Presbyterian Hospital, New York; Merz Pharmaceuticals GmbH (M.A., B.F.-B.), Frankfurt am Main, Germany; Kantar Health (D.M.), Munich, Germany; and Department of Neurology (M.D.B.), Jagiellonian University, Krakow, Poland.

Go to Neurology.org/N for full disclosures. Funding information and disclosures deemed relevant by the authors, if any, are provided at the end of the article. 


\section{Glossary}

$\mathbf{A E}=$ adverse event; $\mathbf{A E S I}=$ adverse event of special interest $\mathbf{B W}=$ body weight $\mathbf{C I}=$ confidence interval; $\mathbf{C P}=$ cerebral palsy; $\mathbf{D Q}=$ drooling quotient; FAS = full analysis set; GICS = Global Impression of Change Scale; IC = informed consent; incoBoNT/ $\mathbf{A}=$ incobotulinumtoxin $\mathrm{A} ; \mathbf{L S}=$ least squares; $\mathbf{M P}=$ main period; $\mathbf{m T D S}=$ modified teacher's drooling scale; $\mathrm{OLEX}=$ open-label extension period; SAE = serious adverse event; $\mathbf{S E S}=$ safety evaluation set; $\mathbf{u S F R}=$ unstimulated salivary flow rate.

\section{Trial Registration Information}

Clinicaltrials.gov: NCT02270736 (clinicaltrials.gov/ct2/show/results/NCT02270736); EU Clinical Trials Register: 2013004532-30 (clinicaltrialsregister.eu/ctr-search/search?query=2013-004532-30).

\section{Classification of Evidence}

This study provides Class I evidence that injection of incobotulinumtoxinA decreases drooling in children aged 6 to 17 years with neurologic disorders.

Sialorrhea (drooling) is a chronic impairment often seen in children with neurodevelopmental disorders or intellectual disability and metabolic and neurodegenerative diseases. Consequences range from quality of life issues to increased morbidity (choking, aspiration, pneumonia). ${ }^{1-3}$ Nonmedicinal treatments include functional therapy and surgery. ${ }^{4}$ Drugs commonly used are anticholinergics (e.g., scopolamine, glycopyrrolate), with typical systemic side effects like bladder retention, chest congestion, and constipation; and botulinum toxin injections to salivary glands. ${ }^{3,5-7}$ Botulinum toxin blocks acetylcholine release, inhibiting secretion of saliva. Several trials have demonstrated efficacy and safety of intraglandular botulinum toxin injections for sialorrhea in adults ${ }^{8-14}$ and children. ${ }^{1,15-26}$

At the time of study planning, the clinical effect of repeated botulinum toxin injections for children had not been tested, optimal doses were undefined, and no studies had fulfilled approval requirements. Most were small, uncontrolled, open-label, or retrospective studies with a single injection cycle, or studies without structured safety monitoring.

IncobotulinumtoxinA (incoBoNT/A; botulinum toxin A free from complexing proteins; Xeomin, Merz Pharmaceuticals $\mathrm{GmbH}$ ) was approved for treatment of sialorrhea in adults in the United States in 2018 and in the European Union in 2019.

This pivotal phase III study investigated efficacy and safety of incoBoNT/A (weight-adapted doses of 20-75 U) compared with placebo for the treatment of chronic sialorrhea associated with neurologic disorders or intellectual disability in children or adolescents. Results from up to 4 consecutive cycles of ultrasound-guided botulinum toxin injections are reported.

\section{Methods}

\section{Standard Protocol Approvals, Registrations, and Patient Consents}

The study was registered in the database of the US National Library of Medicine (clinicaltrials.gov; NCT02270736) and the EU Clinical Trials Register (eudract.ema.europa.eu; number 2013-004532-30) and conducted in accordance with the ethical principles outlined in the Declaration of Helsinki. The protocol, informed consent (IC) forms, and other studyrelated documents were reviewed and approved by local independent ethics committees and institutional review boards. Written IC was obtained from all patients' parents in accordance with regional laws/regulations. All patients were informed to the fullest extent possible about the study, in language and terms they were able to understand. Depending on the extent of the patient's level of understanding and decision-making capacity, the patient assented to the IC given by the patient's parents. Patients of appropriate intellectual maturity personally signed and dated either a separately designed, age-conforming written informed assent form or the written IC if their maturity ensured understanding of the wording.

\section{Study Design}

SIPEXI (Sialorrhea Pediatric Xeomin Investigation) was a prospective, multicenter, phase III study with a randomized, double-blind, parallel-group, placebo-controlled main period (MP) and an open-label extension (OLEX) period. It was conducted from 2015 to 2019 at 27 sites in 6 European Union and non-European Union countries.

Patients were enrolled between February 2015 and December 2017. For safety reasons, patients were recruited sequentially by age: 10- to 17-year-olds first, then 6- to 9-year-olds, then 2to 5-year-olds. A review committee evaluated safety 4 weeks after every 30 randomized patients to detect any issues early. 
Patients aged 6-17 years were randomized (2:1) to receive incoBoNT/A or placebo during the MP, followed by 16 weeks of observation. The 2- to 5-year-olds received active treatment only. Placebo treatment was considered unethical for this group by the authors and the authority approving the trial design (European Medicines Agency) because it required riskbearing analgesia and sedation and because one of the coprimary efficacy parameters (salivary flow) could not be measured in this age group, limiting the value of a placebo control.

Only patients with a clinical need for reinjection after the MP, as assessed by their physicians, were eligible for treatment in the OLEX, receiving up to 3 incoBoNT/A treatments, each with 16 weeks of observation. The study lasted 72 weeks.

Patients assigned to incoBoNT/A received body weight (BW)-dependent total doses of 20-75 U per session. For patients weighing $<30 \mathrm{~kg}$, doses were determined according to a dosing scheme of 5 weight classes, resulting in mean doses between 1.3 and $2.2 \mathrm{U} / \mathrm{kg}$. Patients weighing $\geq 30 \mathrm{~kg}$ received a fixed dose of $75 \mathrm{U}$. The total dose was distributed in a 3:2 ratio among all parotid and submandibular glands (4 injections per session). Patients in the placebo arm received equivalent volumes of placebo solution. All injections were ultrasound-guided. Analgesics and sedatives had to be offered to all patients. Further details of study design and dosing are provided in eMethods, available from Dryad (doi.org/10. 5281/zenodo.5091488).

\section{Study Population}

Children and adolescents ( 2 to 17 years old) included in the study had a neurologic disorder (e.g., cerebral palsy [CP] or traumatic brain injury) or intellectual disability associated with chronic sialorrhea for $\geq 3$ months prior to screening. A modified teacher's drooling scale (mTDS) score of $\geq 6$ ("severe drooling to the extent that clothing becomes damp occasionally"), rated by the investigator, was required. The main exclusion criteria were sialorrhea not related to neurologic disorders or intellectual disability; a $\mathrm{BW}<12 \mathrm{~kg}$; clinically present moderate or severe dysphagia (choking more than once a week), except for patients on parenteral nutrition or nutrition via gastrostomy; pediatric epilepsy not well controlled with antiepileptic drugs; any previous treatment with botulinum toxin for any body region (during the year before screening or within the screening period); and extremely poor dental/oral condition.

\section{Outcome Measures}

The coprimary efficacy endpoints were the change in unstimulated salivary flow rate (uSFR) from study baseline to week 4 and the Carers' Global Impression of Change Scale (GICS) rating at week 4 . Secondary efficacy endpoints were the changes from baseline in uSFR and in GICS ratings at weeks 8 and 12 . The analysis of the coprimary and secondary efficacy endpoints was based on the cohort of 6- to 17-year-olds and compared incoBoNT/A with placebo. Other efficacy endpoints were changes in USFR and GICS ratings at later visits and changes in mTDS ratings and in drooling quotient $\left(\mathrm{DQ}_{i}\right.$ percentage of time a patient drooled) during the MP and OLEX. The uSFR was not determined in 2- to 5-year-olds because the method was considered inappropriate for this group.

The primary safety endpoint was the occurrence of treatmentemergent adverse events (AEs) overall and per injection cycle. Secondary safety endpoints were the occurrence of treatmentemergent AEs of special interest (AESIs) possibly indicative of toxin spread, serious AEs (SAEs), AEs related to treatment, and $\mathrm{AEs}$ leading to discontinuation.

Further details of the outcome assessments are provided in eMethods available from Dryad (doi.org/10.5281/zenodo. 5091488).

\section{Statistical Analysis}

\section{Sample Size Determination}

A total of 219 patients aged 6-17 years were planned to be randomized. Based on a 2:1 randomization, it was estimated that 146 patients in the incoBoNT/A group and 73 patients in the placebo group would provide $95 \%$ power to show a statistically significant difference between incoBoNT/A and placebo for the coprimary endpoints (2-sided $t$ tests, significance level $\alpha=0.05$ ). In addition, the sample size allowed $\geq 100$ patients to be treated with incoBoNT/A and observed for 1 year (anticipating a 30\% dropout rate over 1 year). Furthermore, 30 patients aged $2-5$ years were assumed to be a sufficiently large cohort to generate a safety profile for this age group, which was a regulatory requirement.

\section{Analysis Sets}

Safety analyses were based on the safety evaluation set (SES) of the MP or OLEX, that is, the subset of patients who received study medication. Efficacy analyses were based on the full analysis set (FAS; identical to MP SES) for the MP and on the SES for the OLEX. Long-term analyses focused on patients who received active treatment already during the MP.

\section{Statistical Method}

The confirmatory analysis of the coprimary variables was a mixed model repeated measures (MMRM) analysis (2-sided, significance level $\alpha=0.05$ ) with comparison of least squares (LS) means between incoBoNT/A and placebo, performed on the FAS, limited to patients aged 6-17 years. If both coprimary efficacy variables showed a statistically significant difference vs placebo, the superiority of incoBoNT/A over placebo was considered proven. No $\alpha$-adjustment for multiple testing was necessary. Sensitivity analyses were performed using analysis of covariance models and nonparametric tests. For all efficacy parameters, descriptive statistics were provided. For GICS ratings, response rates (\%) were calculated, with response defined as a GICS rating of $\geq+1$ (at least "minimally improved"). Changes from baseline refer to study baseline. Safety variables were analyzed descriptively. 
Further details of the statistical methods are provided in eMethods available from Dryad (doi.org/10.5281/zenodo. 5091488).

\section{Data Availability}

No individual deidentified patient data are shared. Key elements of the protocol and statistical analysis plan and the main study results are available in the database of the US National Library of Medicine (NCT02270736) and the EU Clinical Trials Register (EudraCT number 2013-004532-30). After regulatory approval in the United States or the European Union in the respective indication and publication of the primary article, Merz will share for 5 years data that relate to the results reported in this article with qualified researchers who provide a valid research question. Prerequisite is a data sharing contract and shared data may only be used for noncommercial purposes. Proposals should be submitted to datasharing@merz.com.

\section{Results}

\section{Study Population}

Overall, 220 patients aged $6-17$ years and 36 patients aged 2-5 years were enrolled. All 6- to 17-year-olds were randomized and treated in the MP (FAS-MP), with 148 patients aged 6-17 years receiving incoBoNT/A and 72 patients aged 6-17 years receiving placebo. Of the 2- to 5-year-olds, 1 patient withdrew before treatment start and 35 patients were treated with incoBoNT/A in a separate, open-label arm of the MP. A total of 214 patients aged 6-17 years and 33 patients aged $2-5$ years were treated in the OLEX (SES-OLEX). Of these, 189 patients aged 6-17 years and all 33 patients aged $2-5$ years completed the OLEX (Figure 1).

Patients' baseline characteristics are summarized in Table 1. The mean baseline uSFR values $(\sim 0.60 \mathrm{~g} / \mathrm{min})$ were within the normal range of saliva production for children. ${ }^{27}$ The mean $\mathrm{mTDS}$ ratings and DQs indicated severe drooling with

Figure 1 Disposition of Patients for the Main Period (MP) and Open-Label Extension (OLEX) of the Study

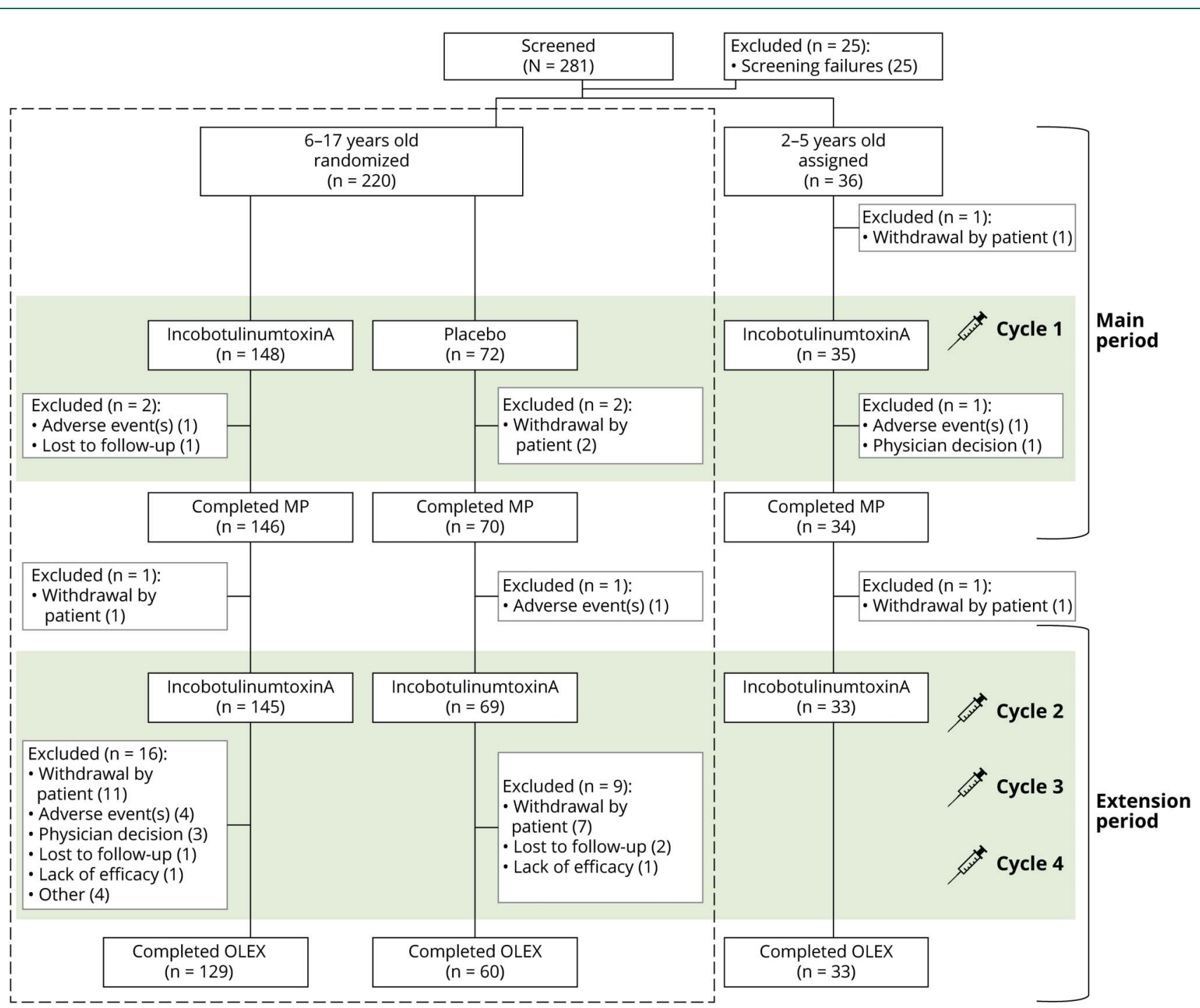

With patient numbers for each treatment arm and phase. Multiple reasons for discontinuation were possible. 
a notable impact on daily life. The incoBoNT/A and placebo groups (6-17 years) were similar with respect to demographics and baseline characteristics.

\section{Treatment Exposure}

During the MP, the 6- to 17-year-old patients received a mean (SD) dose of $1.91(0.25) \mathrm{U} / \mathrm{kg}$, ranging from 1.0 to 2.5 $\mathrm{U} / \mathrm{kg}$. All patients were treated according to the predefined dosing scheme. Considering the numbers of patients per weight group at baseline, this resulted in a median total dose of $40.0 \mathrm{U}$ for patients below $30 \mathrm{~kg}$, with a median dose of 2.0 $\mathrm{U} / \mathrm{kg}$. Patients weighing $\geq 30 \mathrm{~kg}$ received $75 \mathrm{U}$, with a median dose of $1.9 \mathrm{U} / \mathrm{kg}$. The mean doses of the OLEX were similar to those of the MP. For 2 patients, dose reductions were necessary due to AEs (dry mouth and gastric operation) during the 4 th cycle.

In the 2 to 5 years age group, the mean (SD) dose in the MP was $1.70(0.19) \mathrm{U} / \mathrm{kg}$, with a range of 1.4 to $2.1 \mathrm{U} / \mathrm{kg}$. Similar doses were administered throughout the OLEX.

Approximately $90 \%$ of patients received analgesics or sedatives in all injection cycles.

\section{Study Outcomes}

\section{Unstimulated Salivary Flow Rate}

The incoBoNT/A treatment group (age 6-17 years) showed consistently greater decreases in LS-mean uSFR from baseline

Table 1 Patient Demographics and Baseline Disease Characteristics

\begin{tabular}{|c|c|c|c|}
\hline & $\begin{array}{l}\text { Placebo }(6-17 \text { years }) \\
(n=72)\end{array}$ & $\begin{array}{l}\text { IncoBoNT/A (6-17 years) } \\
(n=148)\end{array}$ & $\begin{array}{l}\text { IncoBoNT/A (2-5 years) } \\
(\mathrm{n}=35)\end{array}$ \\
\hline \multicolumn{4}{|l|}{ Demographics } \\
\hline Male & $45(62.5)$ & $93(62.8)$ & $22(62.9)$ \\
\hline Female & $27(37.5)$ & $55(37.2)$ & $13(37.1)$ \\
\hline Age, y & $10.3(3.25)$ & $10.4(3.17)$ & $3.9(0.91)$ \\
\hline $\mathrm{BMI}, \mathrm{kg} / \mathrm{m}^{2}$ & $16.4(3.65)$ & $15.8(3.25)$ & $15.3(1.85)$ \\
\hline \multicolumn{4}{|l|}{ Baseline disease characteristics } \\
\hline Intellectual disability & $64(88.9)$ & $130(87.8)$ & $33(94.3)$ \\
\hline \multicolumn{4}{|l|}{ Primary diagnosis leading to sialorrhea } \\
\hline Cerebral palsy & $43(59.7)$ & $102(68.9)$ & $20(57.1)$ \\
\hline Traumatic brain injury & $1(1.4)$ & $9(6.1)$ & $0(0.0)$ \\
\hline Other ${ }^{a}$ & $28(38.9)$ & $37(25.0)$ & $15(42.9)$ \\
\hline \multicolumn{4}{|l|}{ Baseline assessments } \\
\hline uSFR, g/min & $0.60(0.25)$ & $0.57(0.25)$ & $N A^{b}$ \\
\hline Carers' mTDS & $7.7(1.2)$ & $7.6(1.2)$ & $8.0(1.0)$ \\
\hline Investigators' mTDS & $7.7(1.2)$ & $7.7(1.1)$ & $8.0(1.0)$ \\
\hline DQ & $46.81(25.25)^{c}$ & $42.45(22.32)$ & $45.96(25.85)$ \\
\hline For patients with cerebral palsy: baseline GMFCS E\&R level ${ }^{d}$ & $N=43$ & $N=102$ & $N=20$ \\
\hline I & $3(7.0)$ & $5(4.9)$ & $1(5.0)$ \\
\hline II & $11(25.6)$ & $35(34.3)$ & $6(30.0)$ \\
\hline III & $11(25.6)$ & $11(10.8)$ & $3(15.0)$ \\
\hline IV & $11(25.6)$ & $20(19.6)$ & $1(5.0)$ \\
\hline V & $7(16.3)$ & $31(30.4)$ & $9(45.0)$ \\
\hline
\end{tabular}

Abbreviations: BMI = body mass index; DQ = drooling quotient; GMFCS E\&R = Gross Motor Function Classification System (expanded and revised); incoBoNT / $A=$ incobotulinumtoxinA; $m$ TDS = modified teacher's drooling scale; NA = not available; uSFR = unstimulated salivary flow rate.

Values are $\mathrm{n}(\%)$ or mean (SD).

a "Other diagnoses" comprised a broad range of neurologic conditions, as documented by the treating physicians.

${ }^{b}$ uSFR not measured for 2- to 5-year-olds because method was considered inappropriate for this group.

${ }^{\mathrm{c}}$ Assessment missing for 1 patient.

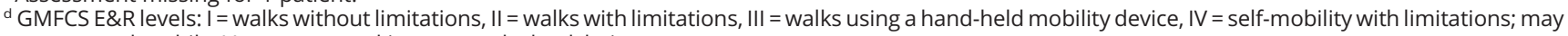
use powered mobile, $\mathrm{V}=$ transported in a manual wheelchair. 
Table 2 Mean Changes in Unstimulated Salivary Flow Rate, g/min

\begin{tabular}{|c|c|c|c|c|c|c|c|}
\hline \multirow[b]{2}{*}{ Week } & \multicolumn{2}{|c|}{ Placebo $(n=72)$} & \multicolumn{2}{|c|}{ IncoBoNT/A $(n=148)$} & \multicolumn{2}{|c|}{ Difference incoBoNT/A vs placebo (MMRM) } & \multirow[b]{2}{*}{$p$ Value } \\
\hline & LS-mean (SE) & $95 \% \mathrm{Cl}$ & LS-mean (SE) & $95 \% \mathrm{Cl}$ & LS-mean (SE) & $95 \% \mathrm{Cl}$ & \\
\hline 4 & $-0.07(0.015)$ & -0.10 to -0.04 & $-0.14(0.012)$ & -0.16 to -0.11 & $-0.06(0.019)$ & -0.10 to -0.03 & 0.0012 \\
\hline 8 & $-0.07(0.015)$ & -0.10 to -0.04 & $-0.16(0.012)$ & -0.18 to -0.13 & $-0.09(0.019)$ & -0.12 to -0.05 & $<0.0001$ \\
\hline 12 & $-0.06(0.016)$ & -0.10 to -0.03 & $-0.16(0.013)$ & -0.19 to -0.14 & $-0.10(0.021)$ & -0.14 to -0.06 & $<0.0001$ \\
\hline 16 & $-0.08(0.015)$ & -0.11 to -0.05 & $-0.15(0.013)$ & -0.18 to -0.12 & $-0.07(0.019)$ & -0.11 to -0.03 & 0.0003 \\
\hline
\end{tabular}

Abbreviations: $\mathrm{Cl}$ = confidence interval; incoBoNT/A = incobotulinumtoxinA.

Mean changes from study baseline to all main period (MP) visits (least squares [LS]-means, adjusted from mixed model repeated measures [MMRM] analysis, 6- to 17-year-olds). Week 4 (4 weeks after MP injection) was the time point of the primary analysis. Data based on full analysis set (6-17 years).

to all time points of the MP, compared to placebo (Table 2). Assessing the change from baseline to week 4 (coprimary analysis), the LS-mean difference between incoBoNT/A and placebo was statistically significant in favor of incoBoNT/A. Secondary analyses (results at week 8 and week 12; Table 2) and further sensitivity analyses confirmed these results.

\section{Carers' GICS Rating During MP}

The incoBoNT/A group (age 6-17 years) showed consistently higher mean carers' GICS ratings at all posttreatment visits of the MP compared to placebo (Figure 2), indicating improvements. Assessing the GICS ratings at week 4 (coprimary analysis), the LS-mean (SE) result for the incoBoNT/A group (0.91 [0.075]; 95\% confidence interval $[\mathrm{CI}][0.76 ; 1.06])$ was notably higher than for the placebo group (0.63 [0.104]; 95\% CI $[0.43 ; 0.84])$. The
LS-mean [SE] difference between the groups was statistically significant $(0.28$ [0.127]; 95\% CI [0.02; 0.53$] ; p=$ 0.032 ). Secondary analyses (results at week 8 and week 12; Figure 2) and further sensitivity analyses confirmed these results.

The analysis of GICS response rates further supported the favorable outcomes. More than $60 \%$ of patients in the incoBoNT/A group (range $70.9 \%$ at week 4 to $63.0 \%$ at week 16) showed a response of at least +1 point on the GICS, compared to $<50 \%$ in the placebo group (range from $47.9 \%$ at week 8 to $34.3 \%$ at week 16 ).

The analysis of both coprimary efficacy variables showed statistically significant improvements, reflecting superiority of incoBoNT/A over placebo for the management of sialorrhea.

Figure 2 Carers' Global Impression of Change Scale (GICS) Mean Ratings at the Main Period (MP) Visits (Active Treatment Compared to Placebo)

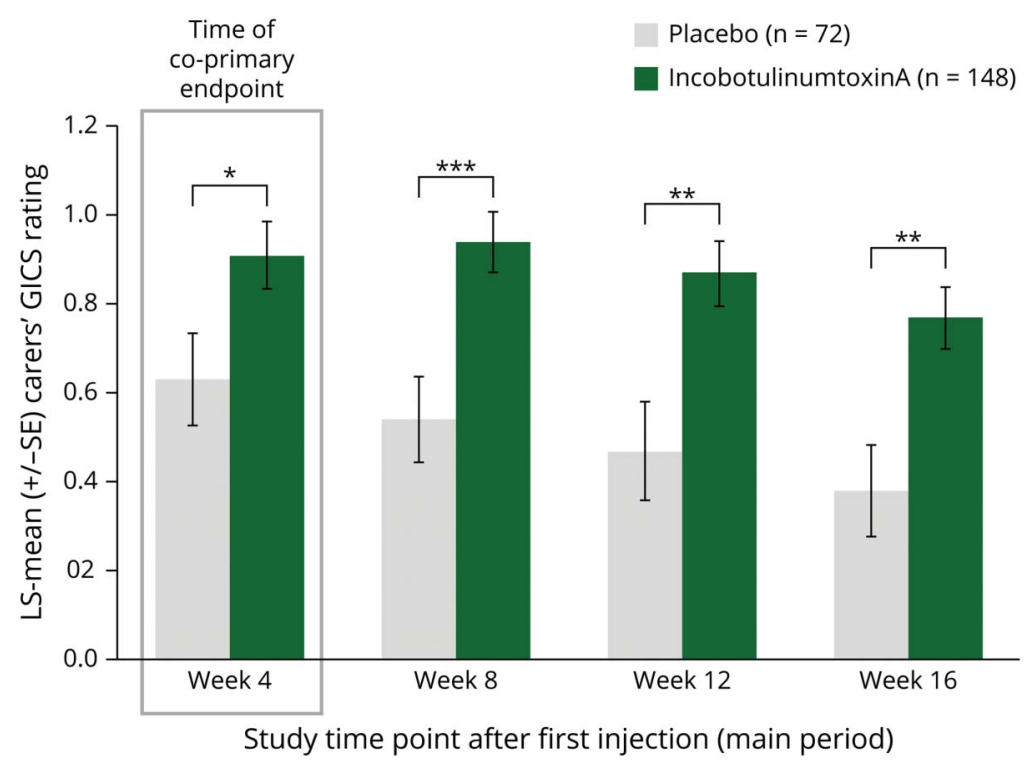

Results for the incobotulinumtoxinA group shown in green placebo in gray. Means are least squares (LS) means (adjusted) from mixed model repeated measures analysis. Week 4 (4 weeks after MP injection) was the time point of the primary analysis. GICS 7-point scale ranges from -3 (very much worse) to +3 (very much improved). Data based on full analysis set (6-17 years). Error bars show SE. ${ }^{*} p<0.05$, ** $p<$ $0.01, * \star \star p \leq 0.001$ 
Figure 3 Unstimulated Salivary Flow Rate (USFR, g/min), Mean Changes From Study Baseline to all Main Period and OpenLabel Extension (OLEX) Period Visits

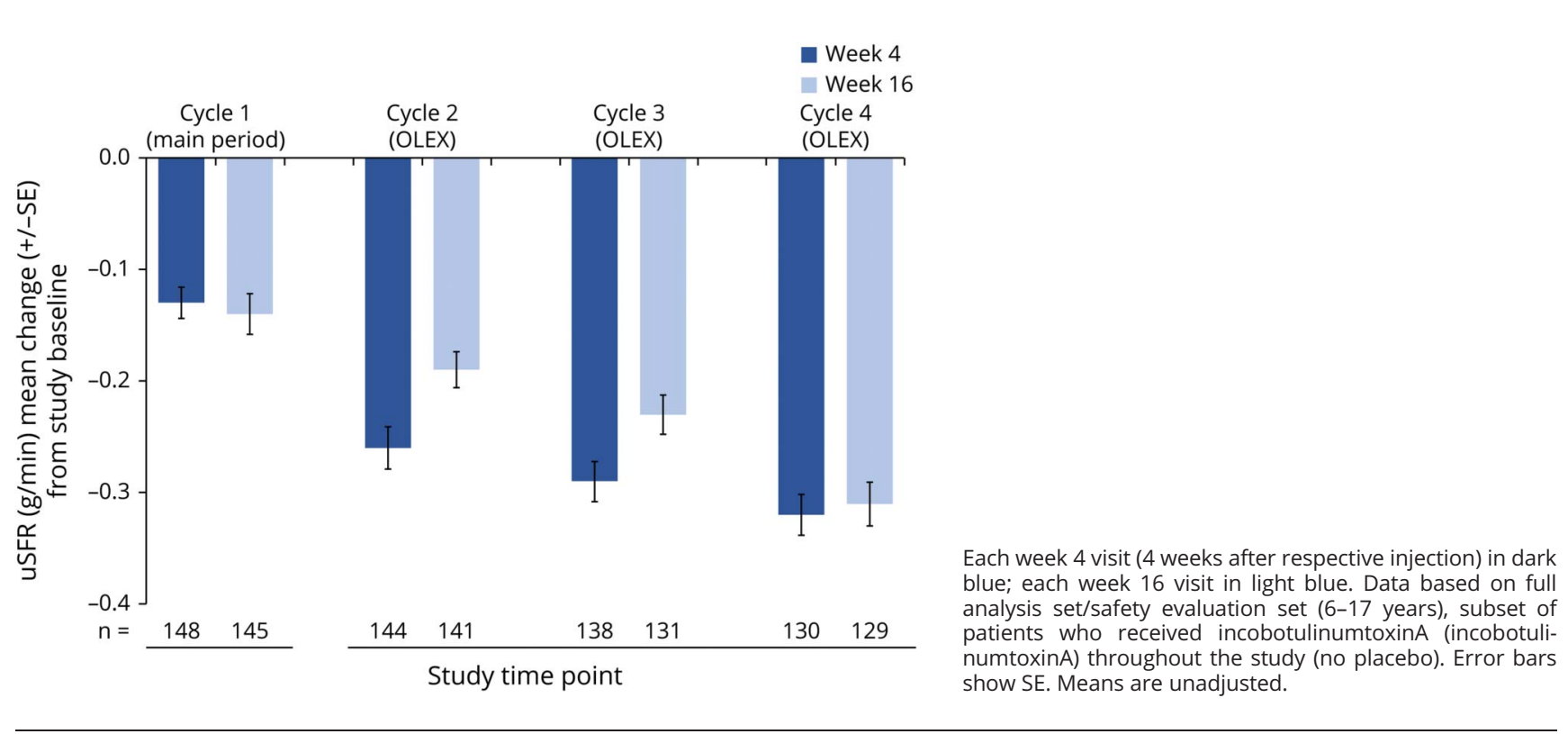

\section{Other Efficacy Assessments}

The carers' and investigators' mTDS ratings and the DQs showed consistently and notably better outcomes for incoBoNT/ A-treated patients than for the placebo group during the MP. In the incoBoNT/A group, the mTDS rating changes from baseline were in the range of -1.5 to -2.0 points on the 9-point scale (mean postbaseline ratings between 5.7 and 6.1 points). The DQ decreases from baseline were between $-11 \%$ and $-14 \%$. For all parameters, the treatment effect appeared to increase from week 4 to week 8 , to remain stable to week 12 , and then to show some decrease to week 16 .

\section{Long-term Efficacy With Repeated Injections}

Repeated treatments with incoBoNT/A showed a prolonged and sustained effect. This was consistently seen in all measures. Mean uSFR decreases from study baseline to week 4 of each treatment cycle became slightly larger with each cycle (Figure 3). Treatment effects were still seen at each week 16

Figure 4 Carers' Global Impression of Change Scale (GICS) Mean Ratings at all Main Period and Open-Label Extension (OLEX) Period Visits

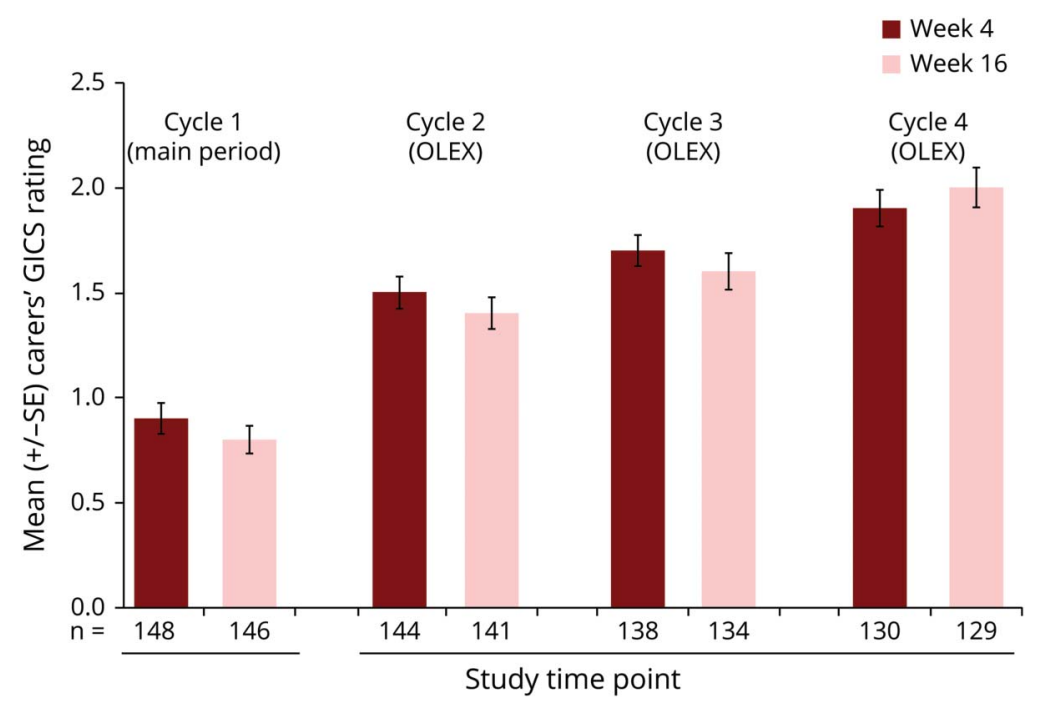

Each week 4 visit ( 4 weeks after respective injection) in dark red; each week 16 visit in light red. GICS 7-point scale from -3 (very much worse) to +3 (very much improved). Data based on full analysis set/safety evaluation set (6-17 years), subset of patients who received incobotulinumtoxinA throughout the study (no placebo). Error bars show SE. Means are unadjusted. 
visit, although less pronounced. Mean GICS ratings were consistently positive, showing an increasing effect over time (Figure 4). The highest ratings around +2 ("much improved") were reached in the 4th cycle. Only small differences in USFR changes and GICS ratings were seen between the respective week 4 and week 16 visits of each cycle, indicating stable improvements.

Consistent improvements compared to baseline were also seen for the mTDS and DQ after repeated injections, with a tendency of gradual increment over time.

\section{Efficacy in 2- to 5-Year-Old Patients}

During the MP, GICS ratings showed notable improvements (mean $[\mathrm{SD}]$ changes ranged between $+1.1[0.8]$ and +1.2 $[0.8]$ ), as did the carers' mTDS results (mean [SD] changes from baseline ranged between -2.0 [1.6] and -2.4 [1.9]). The investigators' mTDS results were similar. DQ outcomes also showed good changes (\%) from baseline (mean [SD] ranged between -16.57 [19.65] and -21.18 [23.32]).

In line with the results for 6- to 17-year-olds, the OLEX outcomes for 2- to 5-year-olds indicated a sustained effect of incoBoNT/A with notable improvements over time. Results were consistent across all measures until the end of the study.

\section{Safety}

\section{AEs in 6- to 17-Year-Old Patients}

During the MP, AE rates were comparable in the 6- to 17-yearold groups (incoBoNT/A: $18.2 \%$ of patients with AEs; placebo: $15.3 \%)$. Rates of SAEs, related AEs, AESIs, and AEs leading to discontinuation were low (Table 3). The only AESI was 1 case of dysphagia (mild, related to treatment, resolved). One patient per group had AEs leading to discontinuation (placebo: pneumonia and epilepsy; incoBoNT/A: nasopharyngitis). No major differences between the groups were seen in the most frequent AEs, mostly respiratory infections.

Of the 148 patients (aged 6-17 years) who received incoBoNT/A during the MP, 145 continued in the OLEX period and $43.4 \%$ (63/145 patients) experienced AEs during the OLEX (Table 3). Most AEs were mild/moderate; most were respiratory tract infections.

SAEs occurred in 8/145 patients (5.5\%) and each individual SAE term was reported in only 1 patient. However, functional gastrointestinal disorder occurred in 2 patients. No SAE was related to treatment and no patient died.

Overall, 8/145 patients (5.5\%) had AEs assessed as related to treatment; none was serious. Among the related AEs, only dysphagia was reported in more than 1 patient ( 4 patients, $2.8 \%$ ) during the OLEX. Dysphagia was the only AESI reported. All 4 patients with dysphagia had single occurrences of the event only (all nonserious, related, mild/moderate, resolved).

AEs leading to discontinuation were documented for 4/145 patients $(2.8 \%)$, and in 1 of these cases the events (dysphagia, saliva altered, choking) were assessed as related to treatment. The incidences of AEs, SAEs, and other important events did not increase notably with increasing number of cycles (first cycle [MP]: 27 patients with AEs

Table 3 Adverse Events (AEs) in the Main Period (MP) and in the Open-Label Extension Period (OLEX) (6- to 17-Year-Olds)

\begin{tabular}{|c|c|c|c|}
\hline & \multirow[b]{2}{*}{ MP, placebo $(n=72)$} & \multicolumn{2}{|c|}{ OLEX (cumulative over 3 cycles) } \\
\hline & & IncoBoNT/A $(n=148)$ & IncoBoNT/A $(n=145)$ \\
\hline AEs & $11(15.3)$ & $27(18.2)$ & $63(43.4)$ \\
\hline AEs related to treatment & $0(0.0)$ & $2(1.4)$ & $8(5.5)$ \\
\hline AESIs & $0(0.0)$ & $1(0.7)$ & $4(2.8)$ \\
\hline AESIs related to treatment & $0(0.0)$ & $1(0.7)$ & $4(2.8)$ \\
\hline SAES & $1(1.4)$ & $0(0.0)$ & $8(5.5)^{a}$ \\
\hline SAEs related to treatment & $0(0.0)$ & $0(0.0)$ & $0(0.0)$ \\
\hline AEs leading to discontinuation & $1(1.4)$ & $1(0.7)$ & $4(2.8)$ \\
\hline AEs leading to discontinuation related to treatment & $0(0.0)$ & $0(0.0)$ & $1(0.7)$ \\
\hline Fatal AEs & $0(0.0)$ & $0(0.0)$ & $0(0.0)$ \\
\hline
\end{tabular}

Abbreviations: AESI = adverse event of special interest; incoBoNT/A = incobotulinumtoxinA; SAE = serious adverse event.

Data based on safety evaluation set (6-17 years). Cumulative OLEX data from safety evaluation set (6-17 years) of patients who had received incoBoNT/A already in the MP. AEs during MP were defined as treatment-emergent with onset or worsening at or after first injection of incoBoNT/A or placebo up to and before first injection of OLEX or, in case of discontinuation before OLEX, up to and including 16 weeks after first injection or last study visit, whichever was later. Values are $\mathrm{n}(\%)$.

${ }^{a}$ MedDRA preferred terms of the SAEs in these patients: functional gastrointestinal disorder (2 patients), gastrointestinal hemorrhage, hematemesis, gastroenteritis rotavirus, influenza, pneumonia, pneumonia bacterial, respiratory tract infection, foreign body in gastrointestinal tract, joint dislocation, anemia, limb deformity, gastric operation. 
[18.2\%]; second cycle: $25 / 145$ patients [17.2\%]; third: $25 /$ 141 patients [17.7\%], fourth: $32 / 131$ patients [24.4\%]). A post hoc analysis showed a median time to onset of AEs after the respective injection of several weeks (63 days [range 1-147 days] in first cycle, 54 days [range 1-123 days] in second cycle, 65 days [range 5-141 days] in third cycle, 84 days [range 16-126 days] in fourth cycle).

In patients who had received placebo in the MP, the overall incidence of AEs in the OLEX (42.0\%; 29/69 patients with $\mathrm{AE}[\mathrm{s}]$ ) was comparable to that in patients who had received incoBoNT/A in the MP. Two patients (2.9\%) from the MP placebo group experienced related AEs during the OLEX. No $\mathrm{SAE}, \mathrm{AESI}, \mathrm{AE}$ leading to discontinuation, or death occurred in this group.

\section{AEs in 2- to 5-Year-Old Patients}

During the MP, $14.3 \%$ of the 2 - to 5 -year-olds (5/35 patients) experienced AEs. One patient (2.9\%) had AEs related to treatment (administration site conditions). Another patient experienced 2 SAEs (staphylococcal bacteremia, generalized tonicclonic seizure [leading to discontinuation], both not related). No $\mathrm{AESI}$ and no death was reported. The only AE that occurred in more than 1 patient was nasopharyngitis (2 patients).

During the OLEX, AEs were documented for 15/33 patients (45.5\%). No related AE, SAE, AESI, AE leading to discontinuation, or death was reported. Most events were respiratory infections. The $\mathrm{AE}$ rate varied between the injection cycles (first cycle $[\mathrm{MP}]: 14.3 \%$; second cycle: $21.2 \%$; third: $15.2 \%$; fourth: $33.3 \%)$.

\section{Other Safety Measures}

Regarding dental/periodontal AEs, oropharyngeal pain was reported for 3 patients $(2.1 \%)$ and oral herpes and dental caries for 2 patients (1.4\%) each, during the OLEX. Among the patients for whom antibody testing was performed, 3 showed positive results at baseline and at study end, but all responded to treatment despite the presence of antibodies against botulinum toxin A. No patient newly developed neutralizing antibodies under therapy.

\section{Classification of Evidence}

This prospective study with a randomized, double-blind, placebo-controlled MP and an OLEX assessed the efficacy and safety of incoBoNT/A (up to $75 \mathrm{U}$ ) for reduction of the salivary flow rate and improvement of chronic sialorrhea in children and adolescents. This study provides Class I evidence that injection of incoBoNT/A decreases drooling in children aged 6-17 years with neurologic disorders.

\section{Discussion}

The SIPEXI study demonstrated that incoBoNT/A was effective and safe for the treatment of sialorrhea in children and adolescents. Management of sialorrhea with incoBoNT/A showed significant and sustained reduction of salivary flow, improving quality of life in 6- to 17 -year-old patients. Both coprimary efficacy endpoints were reached, confirming superiority of incoBoNT/A over placebo. The results were robust and consistent for all measures. The significant decrease in USFR was accompanied by improved quality of life indicators: GICS ratings, mTDS ratings, and DQs. The reduced salivary flow, as seen in the USFR and DQ results, appeared to be clinically meaningful for the patients, easing sialorrhea's effect on everyday life, as seen in the GICS and mTDS results. The mean change seen in the GICS was +0.9 points already at week 4 for the incoBoNT/A group, with $>60 \%$ of patients showing a response of at least +1 point ("minimally improved"). In the OLEX, ratings around +2 ("much improved") were reached. As the maximum attainable improvement on the GICS is +3 , it can be assumed that changes of +1 and +2 are meaningful. This is corroborated by the mTDS results, which provide a more tangible measure of the treatment's effect on daily life. The mean baseline mTDS ratings for the incoBoNT/A group of 6- to 17-year-olds were between 7 points ("severe drooling; clothing becomes damp frequently") and 8 points ("profuse drooling; clothing, hands, and objects become wet occasionally") on the 9-point scale. After 4 weeks, the mean mTDS ratings in this group had already decreased to around 6 points ("severe drooling; clothing becomes damp occasionally"), followed by further improvements to around 3 points ("mild drooling; only lips are wet, but frequently") at the end of the OLEX.

Of note, the analyses of GICS response rates $(\geq+1$ point improvement) also showed a response in the placebo group. However, this was consistently lower than in the incoBoNT/ A group. Placebo responses are well known in developmental disabilities $^{28}$ and might be attributed in this study to expectancy effects (positive expectancy by parents/caregivers, physicians' enthusiasm as the effects of BoNT/A on drooling were already well described in the scientific literature at the time of the study) and to participation effects (raised awareness of health issues and modified behaviors improving health due to study participation). These effects might be larger in children than in adults. ${ }^{29}$ Furthermore, the fact that a treatment effect was detected in the incoBoNT/A as well as in the placebo group proved efficient blinding. The observed placebo effect decreased and the difference to verum increased over time. A study on a comparable pediatric population treated with glycopyrrolate also showed a placebo response for a global efficacy assessment, which was, however, lower than the response to active treatment. ${ }^{30}$

The SIPEXI study results were in line with those from the SIAXI study, a similar trial in adults with sialorrhea, which showed good efficacy and safety for incoBoNT/A (75 U and $100 \mathrm{U})$ compared to placebo, and lasting treatment effects over up to 4 injection cycles. ${ }^{10,11}$

Sialorrhea often requires continuous, long-term treatment. Therefore, SIPEXI investigated the effect of 4 consecutive treatments over more than 1 year. A sustained benefit of repeated treatments became apparent over time. Treatment 
effects became slightly greater with each treatment cycle, indicating a build-up of efficacy. No substantial waning of effect was seen up to 4 months after injection, supporting the notion that up to 3 repeated treatments per year may be sufficient to control sialorrhea.

Comparisons of the SIPEXI results with published literature are limited by differences in study design and endpoints. Furthermore, the published studies of botulinum toxin treatments for pediatric sialorrhea cited here describe the use of onabotulinumtoxinA. The total incoBoNT/A doses employed in SIPEXI (1.3-2.2 U/ kg/session, with a maximum of $75 \mathrm{U}$ for patients with $\mathrm{BW} \geq 30 \mathrm{~kg}$ ) were in the medium range of doses reported for onabotulinumtoxinA.

In the literature, higher doses appear to lead to greater improvements in sialorrhea. ${ }^{16}$ In SIPEXI, the decrease in mean uSFR from baseline to week 4 represents a reduction of around $23 \%$. Even higher decreases in salivary flow of almost 50\% have been reported with higher doses of onabotulinumtoxinA. ${ }^{16}$ However, higher doses also appear to lead to more safety issues. ${ }^{16,31-34}$

DQ results in several published studies were comparable to the present trial. In SIPEXI, the DQ for the incoBoNT/A group decreased by $11 \%-14 \%$ after 1 treatment cycle. This is in line with several smaller studies in children, where similar ranges of $\mathrm{DQ}$ improvements were seen after injections with up to $50 \mathrm{U}$ onabotulinumtoxinA. . $^{24,35,36}$

The SIPEXI results support the concept of a balance between clinically meaningful efficacy and good tolerability and safety by repeated application of medium doses instead of aiming for maximum efficacy with the first injection. The careful accumulation of relevant clinical effects over time may be a valuable treatment scheme, especially for pediatric patients. In SIPEXI, the uSFR only slightly decreased from peak effect at week 4 until the end of cycle visit at week 16 . This difference became smaller from cycle to cycle, providing sustained improvements without exceeding the desired level of salivary flow reduction. Some decrease in effect was observed after several months, but clinical experience suggests that this diminishes with increasing number of injections. It has been reported that chronic use of intraglandular botulinum toxin reduced gland sizes. ${ }^{37}$ A sustained effect of incoBoNT/A after multiple injections could be due to possible atrophy of the glands. It may be worth investigating the causes of the sustained effect with detailed imaging or pathologic studies.

The safety outcomes of SIPEXI were highly encouraging. IncoBoNT/A (20-75 U/kg per session) was shown to be safe and well-tolerated when administered up to 4 times in approximately 1 year, with 16 -week intervals. During the MP, $\mathrm{AE}$ rates were comparable between active treatment and placebo. Overall, AE rates were comparably low, also for SAEs and AEs indicative of potential toxin spread, for example, dysphagia. Over the entire study period, the only types of AEs that accumulated were respiratory infections, which are common in children. This increase was most likely season-related, and the results of active questioning for respiratory AESIs revealed no differences between treatment cycles. In other published studies of botulinum toxin treatment for sialorrhea in children, AEs were often not actively monitored, or the focus was on AEs only, so rates of complications varied widely, but most events were mild. $^{21,23,32,33,38}$

Although a reduced salivary flow could be expected to cause oral health issues, ${ }^{39,40}$ studies addressing changes in salivary composition and flow after botulinum toxin injections did not report a negative effect on patients' oral health. ${ }^{25,41}$ In line with this, in SIPEXI, only few dental/periodontal AEs occurred and there was no evidence of an increase in such AEs with repeated treatments.

Overall, no new or unexpected safety concerns arose, and the high patient retention rate emphasizes tolerability of the treatments. The mandatory ultrasound guidance of injections likely helped minimize AE rates. ${ }^{21}$

The safety results confirm the established notion that botulinum toxins have a favorable safety profile. ${ }^{42-44}$ Anticholinergic drugs like glycopyrrolate and scopolamine, while effective, typically have notably more systemic side effects like behavioral changes, constipation, or mouth dryness. ${ }^{24,44-48}$

In SIPEXI, the efficacy and safety results for the additional cohort of younger patients aged 2-5 years treated with incoBoNT/A were at least as good as those for the older patients, although they tended to receive lower doses per kilogram than the older patients. The efficacy measures consistently showed notable improvements in sialorrhea over the course of the study and AE rates were low.

The sample size for the group of patients aged 2-5 years was small, no placebo control group was included for ethical reasons, and the uSFR was not assessed because the procedure was not suitable for small children. However, the data indicate that incoBoNT/A can be effective and well-tolerated in very young patients.

For the group of patients aged 6-17 years, the SIPEXI results provide strong and robust data. The long-term results may be somewhat biased due to the fixed length of the treatment cycles. Apart from this, the study draws its strengths from the large sample size, the multinational setting, the placebo comparison in the $\mathrm{MP}$, the repeated treatments over 1 year, the almost complete data set with few missing values, and the remarkable patient retention. Particular attention was paid to safety, including safety reviews during recruitment and oral health examinations by a dentist. Furthermore, for the safety and accuracy of procedures, ultrasound-guided injections were mandatory and ultrasound training was provided for the study sites.

The findings presented here show that a conservative, BWdependent dosing approach using 20-75 U incoBoNT/A for repeated treatment of sialorrhea in children can minimize side 
effects while attaining good efficacy and clinically relevant improvements of sialorrhea. This approach is a relevant option for clinicians deciding on optimal individual treatment for children with chronic sialorrhea.

\section{Acknowledgment}

The authors thank the patients and study investigators and the experts who contributed to the regular safety reviews throughout the study: Bernhard Dan (Universite Libre de Bruxelles, Belgium), Antigone Syrigou-Papavasiliou (Pendeli Children's Hospital, Athens, Greece), and Imre Velkey (Borsod County Teaching Hospital, Child Health Center, Miskolc, Hungary).

\section{Study Funding}

This study was funded by Merz Pharmaceuticals GmbH, Frankfurt am Main, Germany.

\section{Disclosure}

S. Berweck was the coordinating investigator in the SIPEXI study and received consultant fees from Ipsen Pharma and speaker fees from Ipsen Pharma and Pharm Allergan. M. Bonikowski was an investigator in the SIPEXI study and an investigator in other Merz, Allergan, Ipsen, and Teva studies; and received research support from Ipsen, Allergan, and Merz and personal fees for consultancy and speaking from Ipsen, Merz, and Allergan. H. Kim was a consultant for Merz and Allergan; received research grants from Ipsen, TEVA, and Allergan; was a pediatric spasticity advisory member for Allergan and Ipsen; and received honoraria from Catalyst Medical Education LLC, AiCME Medical Education, and American Academy of Physical Medicine \& Rehabilitation. M. Althaus is an employee of Merz Pharmaceuticals GmbH. B. Flatau-Baqué is an employee of Merz Pharmaceuticals GmbH. D. Mueller is a medical writer employed at Kantar Health, Munich, Germany. M. Banach was an investigator in the SIPEXI study, participated in the advisory board and speaker bureau for Merz, and served as a consultant and speaker for Ipsen, Allergan, Kedrion, and Shire. Go to Neurology.org/N for full disclosures.

\section{Publication History}

Received by Neurology November 3, 2020. Accepted in final form July 6, 2021.

\section{Appendix Authors}

\begin{tabular}{lll}
\hline Name & Location & Contribution \\
\hline $\begin{array}{l}\text { Steffen } \\
\text { Berweck, } \\
\text { MD }\end{array}$ & $\begin{array}{l}\text { Schön Klinik, Vogtareuth, } \\
\text { Germany and Dr. von } \\
\text { Hauner Children's Hospital, } \\
\text { Ludwig-Maximilians- } \\
\text { University, Munich, Germany }\end{array}$ & $\begin{array}{l}\text { Study concept and design, } \\
\text { acquisition, analysis and } \\
\text { interpretation of study data, } \\
\text { critical revision of } \\
\text { manuscript for important } \\
\text { intellectual content }\end{array}$ \\
\hline $\begin{array}{l}\text { Marcin } \\
\text { Bonikowski, } \\
\text { MD, PhD }\end{array}$ & $\begin{array}{l}\text { Mazovian Neuropsychiatry } \\
\text { Poland }\end{array}$ & $\begin{array}{l}\text { Acquisition and } \\
\text { interpretation of study data, } \\
\text { critical revision of }\end{array}$ \\
& & $\begin{array}{l}\text { manuscript for important } \\
\text { intellectual content }\end{array}$
\end{tabular}

Appendix (continued)

\begin{tabular}{|c|c|c|}
\hline Name & Location & Contribution \\
\hline $\begin{array}{l}\text { Heakyung } \\
\text { Kim, MD }\end{array}$ & $\begin{array}{l}\text { Pediatric Rehabilitation } \\
\text { Medicine at the Department } \\
\text { of Rehabilitation \& } \\
\text { Regenerative Medicine of } \\
\text { Columbia University Irving } \\
\text { Medical Center/New York } \\
\text { Presbyterian Hospital, New } \\
\text { York }\end{array}$ & $\begin{array}{l}\text { Critical revision of } \\
\text { manuscript for important } \\
\text { intellectual content }\end{array}$ \\
\hline $\begin{array}{l}\text { Michael } \\
\text { Althaus, MD, } \\
\text { FFPM }\end{array}$ & $\begin{array}{l}\text { Merz Pharmaceuticals } \\
\text { GmbH, Frankfurt am Main, } \\
\text { Germany }\end{array}$ & $\begin{array}{l}\text { Study concept and design, } \\
\text { acquisition, analysis and } \\
\text { interpretation of study data, } \\
\text { critical revision of } \\
\text { manuscript for important } \\
\text { intellectual content }\end{array}$ \\
\hline $\begin{array}{l}\text { Birgit } \\
\text { Flatau- } \\
\text { Baqué }\end{array}$ & $\begin{array}{l}\text { Merz Pharmaceuticals } \\
\text { GmbH, Frankfurt am Main, } \\
\text { Germany }\end{array}$ & $\begin{array}{l}\text { Study concept and design, } \\
\text { acquisition, analysis and } \\
\text { interpretation of study data, } \\
\text { critical revision of } \\
\text { manuscript for important } \\
\text { intellectual content }\end{array}$ \\
\hline $\begin{array}{l}\text { Daniela } \\
\text { Mueller, } \\
\text { PhD }\end{array}$ & $\begin{array}{l}\text { Kantar Health, Munich, } \\
\text { Germany }\end{array}$ & $\begin{array}{l}\text { Drafting and critical revision } \\
\text { of manuscript for important } \\
\text { intellectual content }\end{array}$ \\
\hline $\begin{array}{l}\text { Marta } \\
\text { Banach, MD, } \\
\text { PhD }\end{array}$ & $\begin{array}{l}\text { Jagiellonian University, } \\
\text { Krakow, Poland }\end{array}$ & $\begin{array}{l}\text { Study concept and design, } \\
\text { acquisition, analysis and } \\
\text { interpretation of study data, } \\
\text { critical revision of } \\
\text { manuscript for important } \\
\text { intellectual content }\end{array}$ \\
\hline
\end{tabular}

\section{References}

1. Gubbay A, Blackmore MA. Effects of salivary gland botulinum toxin-A on drooling and respiratory morbidity in children with neurological dysfunction. Int J Pediatr Otorhinolaryngol. 2019;124:124-128.

2. Erasmus CE, van Hulst K, Rotteveel JJ, Willemsen MA, Jongerius PH. Clinical practice: swallowing problems in cerebral palsy. Eur J Pediatr. 2012;171(3):409-414.

3. Daniel SJ. Multidisciplinary management of sialorrhea in children. Laryngoscope. 2012;122(suppl 4):S67-S68.

4. Lawrence R, Bateman N. Surgical management of the drooling child. Curr Otorhinolaryngol Rep. 2018;6(1):99-106.

5. Walshe M, Smith M, Pennington L. Interventions for drooling in children with cerebral palsy. Cochrane Database Syst Rev. 2012;11:CD008624.

6. Morgante F, Bavikatte G, Anwar F, Mohamed B. The burden of sialorrhoea in chronic neurological conditions: current treatment options and the role of incobotulinumtoxinA (Xeomin(R)). Ther Adv Neurol Disord. 2019;12:1756286419888601.

7. Fairhurst CB, Cockerill H. Management of drooling in children. Arch Dis Child Educ Pract Ed. 2011;96(1):25-30.

8. Restivo DA, Panebianco M, Casabona A, et al. Botulinum toxin A for sialorrhoea associated with neurological disorders: evaluation of the relationship between effect of treatment and the number of glands treated. Toxins (Basel). 2018;10(2):55.

9. Isaacson $\mathrm{SH}$, Ondo W, Jackson CE, et al. Safety and efficacy of rimabotulinumtoxin B for treatment of sialorrhea in adults: a randomized clinical trial. JAMA Neurol. 2020; $77(4): 461-469$.

10. Jost WH, Friedman A, Michel O, et al. SIAXI: placebo-controlled, randomized, double-blind study of incobotulinumtoxinA for sialorrhea. Neurology. 2019;92(17): e1982-e1991.

11. Jost WH, Friedman A, Michel O, et al. Long-term incobotulinumtoxinA treatment for chronic sialorrhea: efficacy and safety over 64 weeks. Parkinsonism Relat Disord. 2020; 70:23-30.

12. Gonzalez LM, Martinez C, Bori YFI, Suso-Vergara S. Factors in the efficacy, safety, and impact on quality of life for treatment of drooling with botulinum toxin type A in patients with cerebral palsy. Am J Phys Med Rehabil. 2017;96(2):68-76.

13. Martinez-Poles J, Nedkova-Hristova V, Escribano-Paredes JB, et al. Incobotulinumtoxin A for sialorrhea in neurological disorders: a real-life experience. Toxins. $2018 ; 10(6): 217$.

14. Lipp A, Trottenberg T, Schink T, Kupsch A, Arnold G. A randomized trial of botulinum toxin A for treatment of drooling. Neurology. 2003;61(9):1279-1281.

15. Reid SM, Johnstone BR, Westbury C, Rawicki B, Reddihough DS. Randomized trial of botulinum toxin injections into the salivary glands to reduce drooling in children with neurological disorders. Dev Med Child Neurol. 2008;50(2):123-128. 
16. Moller E, Pedersen SA, Vinicoff PG, et al. Onabotulinumtoxin A treatment of drooling in children with cerebral palsy: a prospective, longitudinal open-label study. Toxins. 2015;7(7):2481-2493

17. Rodwell K, Edwards P, Ware RS, Boyd R. Salivary gland botulinum toxin injections for drooling in children with cerebral palsy and neurodevelopmental disability: a systematic review. Dev Med Child Neurol. 2012;54(11):977-987.

18. Van Hulst K, Van Der Burg JJ, Jongerius PH, Geurts AC, Erasmus CE. Changes in severity and impact of drooling after submandibular gland botulinum neurotoxin A injections in children with neurodevelopmental disabilities. Dev Med Child Neurol. 2020;62(3):354-362.

19. Alrefai AH, Aburahma SK, Khader YS. Treatment of sialorrhea in children with cerebral palsy: a double-blind placebo controlled trial. Clin Neurol Neurosurg. 2009; 111(1):79-82.

20. Banerjee KJ, Glasson C, O’Flaherty SJ. Parotid and submandibular botulinum toxin A injections for sialorrhoea in children with cerebral palsy. Dev Med Child Neurol. 2006; 48(11):883-887.

21. Lungren MP, Halula S, Coyne S, Sidell D, Racadio JM, Patel MN. Ultrasound-guided botulinum toxin type A salivary gland injection in children for refractory sialorrhea: 10-year experience at a large tertiary children's hospital. Pediatr Neurol. 2016;54: $70-75$.

22. Schroeder AS, Kling T, Huss K, et al. Botulinum toxin type A and B for the reduction of hypersalivation in children with neurological disorders: a focus on effectiveness and therapy adherence. Neuropediatrics. 2012;43(1):27-36.

23. Taib BG, Williams SP, Sood S, Ung K, Nixon PP, Sharma R. Treatment of sialorrhoea with repeated ultrasound-guided injections of botulinum toxin $\mathrm{A}$ into the parotid and submandibular glands. Br J Oral Maxillofac Surg. 2019;57(5):442-448.

24. Jongerius PH, van den Hoogen FJ, van Limbeek J, Gabreels FJ, van Hulst K, Rotteveel $\mathrm{JJ}$. Effect of botulinum toxin in the treatment of drooling: a controlled clinical trial. Pediatrics. 2004;114(3):620-627.

25. Wu KP, Ke JY, Chen CY, Chen CL, Chou MY, Pei YC. Botulinum toxin type A on oral health in treating sialorrhea in children with cerebral palsy: a randomized, doubleblind, placebo-controlled study. J Child Neurol. 2011;26(7):838-843.

26. Wilken B, Aslami B, Backes H. Successful treatment of drooling in children with neurological disorders with botulinum toxin A or B. Neuropediatrics. 2008;39:200-204.

27. Forcella L, Filippi C, Waltimo T, Filippi A. Measurement of unstimulated salivary flow rate in healthy children aged 6 to 15 years. Swiss Dent J. 2018;128(12):962-967.

28. Sandler A. Placebo effects in developmental disabilities: implications for research and practice. Ment Retard Dev Disabil Res Rev. 2005;11(2):164-170.

29. Rheims S, Cucherat M, Arzimanoglou A, Ryvlin P. Greater response to placebo in children than in adults: a systematic review and meta-analysis in drug-resistant partial epilepsy. PLoS Med. 2008;5(8):e166.

30. Zeller RS, Lee HM, Cavanaugh PF, Davidson J. Randomized phase III evaluation of the efficacy and safety of a novel glycopyrrolate oral solution for the management of chronic severe drooling in children with cerebral palsy or other neurologic conditions. Ther Clin Risk Manag. 2012;8:15-23.

31. Khan WU, Campisi P, Nadarajah S, et al. Botulinum toxin A for treatment of sialorrhea in children: an effective, minimally invasive approach. Arch Otolaryngol Head Neck Surg. 2011;137(4):339-344.
32. Chan KH, Liang C, Wilson P, Higgins D, Allen GC. Long-term safety and efficacy dat on botulinum toxin type A: an injection for sialorrhea. JAMA Otolaryngol Head Neck Surg. 2013;139(2):134-138.

33. van Hulst $\mathrm{K}$, Kouwenberg CV, Jongerius $\mathrm{PH}$, et al. Negative effects of submandibular botulinum neurotoxin A injections on oral motor function in children with drooling due to central nervous system disorders. Dev Med Child Neurol. 2017;59(5):531-537.

34. Nordgarden H, Osterhus I, Moystad A, et al. Drooling: are botulinum toxin injections into the major salivary glands a good treatment option? J Child Neurol. 2012;27(4): 458-464.

35. Erasmus CE, Van Hulst K, Van Den Hoogen FJ, et al. Thickened saliva after effective management of drooling with botulinum toxin A. Dev Med Child Neurol. 2010;52(6) e114-118.

36. Scheffer AR, Erasmus C, van Hulst K, van Limbeek J, Jongerius PH, van den Hoogen FJ. Efficacy and duration of botulinum toxin treatment for drooling in 131 children. Arch Otolaryngol Head Neck Surg. 2010;136(9):873-877.

37. Cardona I, Saint-Martin C, Daniel SJ. Effect of recurrent onabotulinum toxin A injection into the salivary glands: an ultrasound measurement. Laryngoscope. 2015; 125(10):E328-E332.

38. Suskind DL, Tilton A. Clinical study of botulinum-A toxin in the treatment of sialorrhea in children with cerebral palsy. Laryngoscope. 2002;112(1):73-81.

39. Scully C, Felix DH. Oral medicine: update for the dental practitioner: dry mouth and disorders of salivation. Br Dent J. 2005;199(7):423-427.

40. Ferraz Dos Santos B, Dabbagh B, Daniel SJ, Schwartz S. Association of onabotulinum toxin A treatment with salivary $\mathrm{pH}$ and dental caries of neurologically impaired children with sialorrhea. Int J Paediatr Dent. 2016;26(1):45-51.

41. Tiigimae-Saar J, Tamme T, Rosenthal M, Kadastik-Eerme L, Taba P. Saliva changes in Parkinson's disease patients after injection of Botulinum neurotoxin type A. Neurol Sci. 2018;39(5):871-877.

42. Bekkers S, Delsing CP, Kok SE, et al. Randomized controlled trial comparing botulinum vs surgery for drooling in neurodisabilities. Neurology. 2019;92(11) e1195-e1204.

43. Lakraj AA, Moghimi N, Jabbari B. Sialorrhea: anatomy, pathophysiology and treat ment with emphasis on the role of botulinum toxins. Toxins. 2013;5(5):1010-1031.

44. Jongerius PH, Rotteveel JJ, van Limbeek J, Gabreels FJ, van Hulst K, van den Hoogen FJ. Botulinum toxin effect on salivary flow rate in children with cerebral palsy. Neurology. 2004;63(8):1371-1375.

45. Eiland LS. Glycopyrrolate for chronic drooling in children. Clin Ther. 2012;34(4): 735-742.

46. Zeller RS, Davidson J, Lee HM, Cavanaugh PF. Safety and efficacy of glycopyrrolate oral solution for management of pathologic drooling in pediatric patients with cerebral palsy and other neurologic conditions. Ther Clin Risk Manag. 2012;8:25-32.

47. Parr JR, Todhunter E, Pennington L, et al. Drooling Reduction Intervention randomised trial (DRI): comparing the efficacy and acceptability of hyoscine patches and glycopyrronium liquid on drooling in children with neurodisability. Arch Dis Child. 2018;103(4):371-376.

48. Mier RJ, Bachrach SJ, Lakin RC, Barker T, Childs J, Moran M. Treatment of sialorrhea with glycopyrrolate: a double-blind, dose-ranging study. Arch Pediatr Adolesc Med. 2000;154(12):1214-1218. 


\section{Neurology}

\section{Placebo-Controlled Clinical Trial of IncobotulinumtoxinA for Sialorrhea in Children: SIPEXI}

Steffen Berweck, Marcin Bonikowski, Heakyung Kim, et al. Neurology 2021;97;e1425-e1436 Published Online before print August 2, 2021

DOI 10.1212/WNL.0000000000012573

This information is current as of August 2, 2021

Updated Information \& Services

References

Subspecialty Collections

Permissions \& Licensing

Reprints including high resolution figures, can be found at: http://n.neurology.org/content/97/14/e1425.full

This article cites 48 articles, 5 of which you can access for free at: http://n.neurology.org/content/97/14/e1425.full\#ref-list-1

This article, along with others on similar topics, appears in the following collection(s):

All Pediatric

http://n.neurology.org/cgi/collection/all_pediatric

Botulinum toxin

http://n.neurology.org/cgi/collection/botulinum_toxin

Class I

http://n.neurology.org/cgi/collection/class_1

Clinical trials Randomized controlled ( $\overline{\mathbf{C}}$ ONSORT agreement)

http://n.neurology.org/cgi/collection/clinical_trials_randomized_contro lled_consort_agreement

Information about reproducing this article in parts (figures,tables) or in its entirety can be found online at:

http://www.neurology.org/about/about_the_journal\#permissions

Information about ordering reprints can be found online:

http://n.neurology.org/subscribers/advertise

Neurology ${ }^{\circledR}$ is the official journal of the American Academy of Neurology. Published continuously since 1951, it is now a weekly with 48 issues per year. Copyright Copyright ( 2021 The Author(s). Published by Wolters Kluwer Health, Inc. on behalf of the American Academy of Neurology.. All rights reserved. Print ISSN: 0028-3878. Online ISSN: 1526-632X.

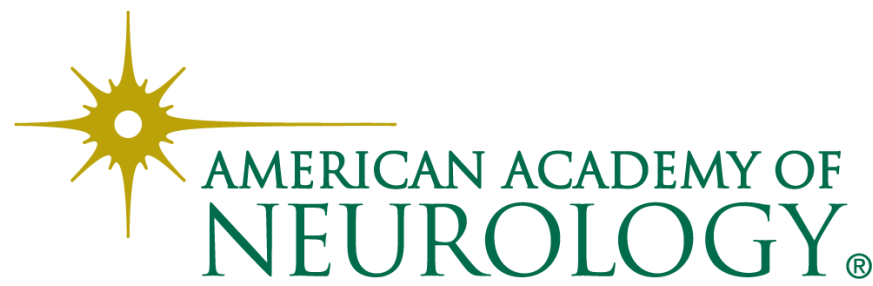

